Proceedings of the 1997 IEEE

International Conference on Robotics and Automation

Albuquerque, New Mexico - April 1997

\title{
Kinematic Design of a Six Degree-of-Freedom In-Parallel Manipulator for Probing Task
}

\author{
J.H. Shim*, J.Y. Park*, D.S. Kwon**, H.S. Cho**, and S. Kim*** \\ *Department of Automation and Design Engineering, KAIST, Seoul, Korea \\ **Department of Mechanical Engineering, KAIST, Taejon, Korea \\ ***Production Engineering Center, Samsung Electronics, Ltd., Suwon, Korea
}

\begin{abstract}
This paper presents a kinematic analysis of a parallel manipulator we have been developing for the probing task application that requires high bandwidth, active compliance, and high precision. Based on the concept of macro/micro manipulators, the parallel manipulator serves as a wrist of a macro manipulator. The developed manipulator is a class of in-parallel platforms with 3 PRPS( prismatic-revolute-prismatic-spherical joints ) chain geometry. The main advantages of this manipulator, compared with the typical Stewart platform type, are the capability of pure rotation generation and the easy prediction of the moving platform motion. The purpose of this paper is to develope an efficient kinematic model which can be used for real-time control and to propose systematic methods to design the manipulator considering workspace, manipulability, resistivity, and the existence conditions of the forward kinematic solution. A series of simulation are carried out to show the kinematic characteristics and performance of the mechanism. A prototype manipulator has been built based on the kinematic design analysis.
\end{abstract}

\section{Introduction}

In the past two decades, there have been considerable developments in the area of parallel manipulators. Obviously, parallel manipulators offer high structural stiffness and precise positioning accuracy over serial ones.

Parallel manipulators have been studied by many researchers, usually in the configuration known as a Stewart platform[1-5]. The Stewart platform was originally designed as an aircraft simulator[1]. Since then, various applications of the Stewart platform have been investigated such as a compliance device for assembly[2], a force/torque sensor[3], an active vibration isolator[4], and a master hand mechanism for teleoperation[5]. But in general, the Stewart platform has some disadvantages that forward kinematics is too complex to solve in real-time and the prediction of the motion of each joint is not intuitive.

Other configurations for parallel manipulators with six degree-of-freedom have also been proposed. Behi[6] developed a configuration with three legs where each leg consists of a PRPS chain. Hudgens and Tesar[7] investigated a device with six inextensible legs where each leg is driven by a four-bar mechanism mounted on the base platform. Alizade et al.[8] proposed a parallel manipulator which has three legs, mounted on moving sliders passing through a circular trajectory. Previous works in these modified Stewart platform manipulators have shown few industrial applications compared with the Stewart platform.

Most tasks carried out by robotic manipulators are involved with the interaction with the environment such as precision assembly, machining, and probing operation. As the contact occurs very fast, limited control bandwidth of conventional robot controllers restricts their active force control. Concept of the macro/micro manipulator has been proposed as one of the methods to solve the contact problem[9]. A micro manipulator is attached to a macro conventional robot serially. The micro manipulator, generally a specialzed device in the form of fingers or a wrist, is well adapted to active force control due to its low inertia. Some of the micro manipulator has parallel structures[10]. Most of their structures are based on the Stewart platform. The actuators of the manipulators have been electrical rams and pneumatic actuators.

This paper presents a kinematic analysis of a parallel manipulator we have been developing for industrial applications that require high control bandwidth, active compliance, and high precision as shown in Fig. 1. The developed manipulator is a class of in-parallel platforms with 3 PRPS chains geometry whose design is based on the Behi mechanism[8]. The main advantages of this 
manipulator, compared with the Stewart platform type, are the capability to produce pure rotation and to predict the motion of the moving platform intuitively. Also, this manipulator has simple kinematic characteristics compared with the Stewart platform. Therefore, controlling in real-time is possible due to less computational burden. Although the basic structure of the developed mechanism is similar to Behi platform, this paper emphasizes the following contents that have not been addressed by others: a simple kinematic model of the 3 PRPS type parallel manipulator which can be used for real-time control and systematic methods to design the manipulator considering workspace, manipulability, resistivity, and the existence range of the forward kinematic solution.

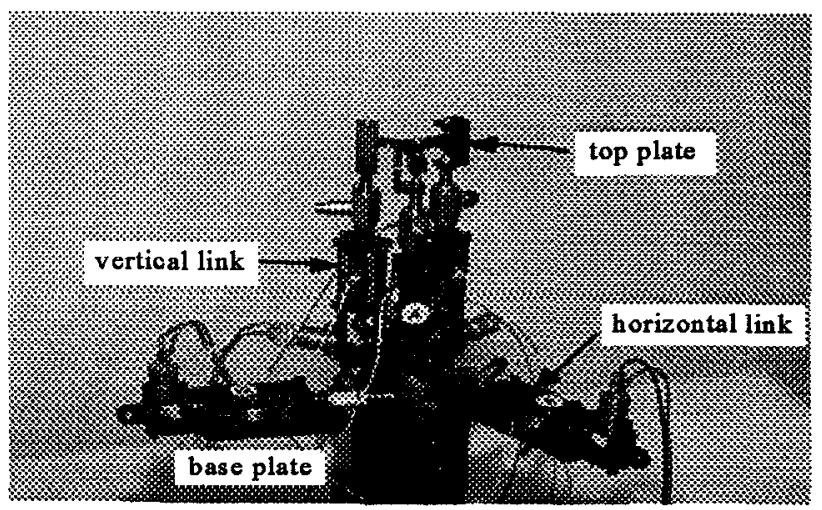

Fig. 1. A photograph of the proposed parallel manipulator

\section{Inverse and Forward Kinematics}

The developed mechanism consists of a base plate, a top plate, three horizontal actuating links, and three vertical actuating links. The three horizontal links provide three degree-of-freedom, that are one degree of orientational freedom and two degrees of translational freedom. The three vertical links provide three degreeof-freedom, that are two degrees of orientational freedom and one degree of translational freedom.

The notation used to describe the kinematics of the proposed mechanism is shown in Fig. 2. The fixed global coordinates called the base frame $(X, Y, Z)$ is located at the center of the mass of the base plate. Another reference coordinate, called the top frame ( $x$, $y, z$ ), is located at the center of the mass of the top plate. The coordinate $(x, y, z)$ with respect to the base frame $(X, Y, Z$ ) can be described by the vectors of the homogeneous transformation matrix $T$ :

$$
{ }_{o}^{\prime} T=(\vec{n}, \vec{o}, \vec{a}, \vec{p})=\left[\begin{array}{cccc}
n_{1} & a_{1} & a_{1} & X_{c} \\
n_{2} & a_{2} & a_{2} & Y_{c} \\
n_{3} & a_{3} & a_{3} & Z_{c} \\
0 & 0 & 0 & 1
\end{array}\right]
$$

where $(\vec{n}, \vec{o}, \vec{a})$ and $\left(X_{c}, Y_{\mathrm{c}}, Z_{\mathrm{c}}\right)$ are the orientation vector and the position of the top platform center with respect to the base frame ( $X, Y, Z$ ), respectively. The top plate is connected to the vertical links with ball joints $B_{\mathrm{i}}$ which are equally spaced at 120 degrees and at a radius $r$ from the center of the top platform. The other ends of the vertical links are connected to the horizontal links through equally spaced pin joints $P_{\mathrm{i}}$ at a radius $R$ from the center of the base platfrom.

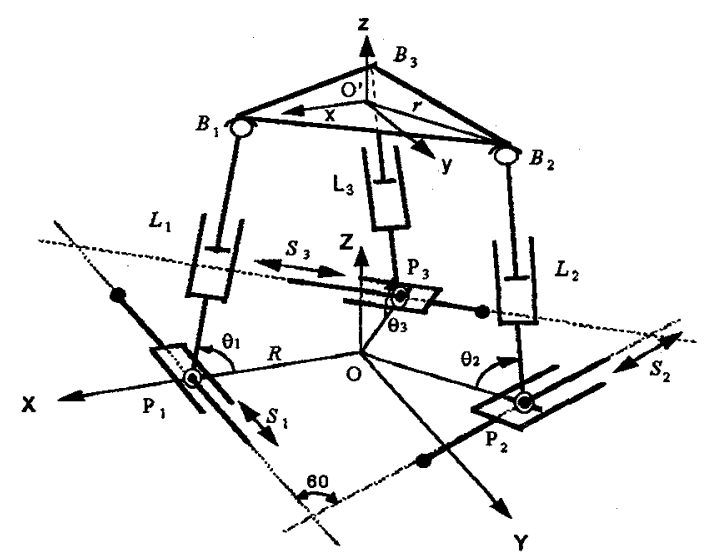

Fig. 2. Kinematic structure with coordinate assignment

The inverse kinematics problem can be briefly stated as: for a given position and orientation of the top platform, compute the actuating length of eack link. From the relationship that the links $P_{1} B_{1}, P_{2} B_{2}$, and $P_{3} B_{3}$ are constrained by the pin joints, we can obtain the actuating lengths of the horizontal links $S_{\mathrm{i}}$ as follows:

$$
\begin{aligned}
& S_{1}=n_{2} r+Y_{C} \\
& S_{2}=\frac{\sqrt{3}}{4} n_{1} r+\frac{1}{4} n_{2} r-\frac{3}{4} o_{1} r-\frac{\sqrt{3}}{4} o_{2} r-\frac{\sqrt{3}}{2} X_{C}-\frac{1}{2} Y_{C} \\
& S_{3}=-\frac{\sqrt{3}}{4} n_{1} r+\frac{1}{4} n_{2} r-\frac{3}{4} o_{1} r+\frac{\sqrt{3}}{4} o_{2} r+\frac{\sqrt{3}}{2} X_{C}-\frac{1}{2} Y_{C}
\end{aligned}
$$

Then, the lengths of the vertical links $L_{\mathrm{i}}$ can be obtained from the the following vector analysis:

$$
\overline{O O^{\prime}}+\overline{O^{\prime} B_{i}}=\overline{O P_{i}}+\overline{P_{i} B_{i}} \quad \text { for } i=1,2,3 \text {. }
$$




$$
\begin{aligned}
L_{1}^{2}= & X_{C}{ }^{2}+Y_{C}{ }^{2}+Z_{C}{ }^{2}+r^{2}+R^{2}+S_{1}^{2}-2 R X_{C}-2 S_{1} Y_{C} \\
& +2 m_{1}\left(X_{C}-R\right)+2 m_{2}\left(Y_{C}-S_{1}\right)+2 m_{3} Z_{C} \\
L_{2}{ }^{2}= & X_{C}{ }^{2}+Y_{C}{ }^{2}+Z_{C}{ }^{2}+r^{2}+R^{2}+S_{2}^{2}+R X_{C}-\sqrt{3} R Y_{C} \\
& +\sqrt{3} S_{2} X_{C}+S_{2} Y_{C}+\left(X_{C}+\frac{1}{2} R+\frac{\sqrt{3}}{2} S_{2}\right)\left(\sqrt{3} o_{1}-n_{1}\right) r \\
& +\left(Y_{C}-\frac{\sqrt{3}}{2} R+\frac{1}{2} S_{2}\right)\left(\sqrt{3} o_{2}-m_{2}\right) r+\left(\sqrt{3} o_{3}-n_{3}\right) r Z_{C} \\
L_{3}{ }^{2}= & X_{C}{ }^{2}+Y_{C}{ }^{2}+Z_{C}{ }^{2}+r^{2}+R^{2}+S_{3}^{2}+R X_{C}+\sqrt{3} R Y_{C}-\sqrt{3} S_{3} X_{C} \\
& +S_{3} Y_{C}-\left(X_{C}+\frac{1}{2} R-\frac{\sqrt{3}}{2} S_{3}\right)\left(\sqrt{3} o_{1}+n_{1}\right) r \\
& -\left(Y_{C}+\frac{\sqrt{3}}{2} R+\frac{1}{2} S_{3}\right)\left(\sqrt{3} o_{2}+n_{2}\right) r-\left(\sqrt{3} o_{3}+n_{3}\right) r Z_{C}
\end{aligned}
$$

If the position of the top plate is given, the solutions of the inverse kinematics are uniquely determined as shown in (1) and (3).

The forward kinematics problem can be stated as: for given actuating lengths of the extensible links, compute the position $\left(X_{\mathrm{c}}, Y_{\mathrm{c}}, Z_{\mathrm{c}}\right)$ and orientation vector $(\vec{n}, \vec{o}, \vec{a})$ of the top platform. The angles $\theta_{1}$ are defined to be the angles between the base platform and the vertical links $P_{\mathrm{i}} B_{\mathrm{i}}$ ( for $i=1,2,3$ ). Since the distance between any two adjacent ball joints is $\sqrt{3} r$, the vector relations can be expressed as:

$$
\begin{aligned}
& \overrightarrow{O P_{i}}+{\overrightarrow{P_{i} B_{i}}}_{\vec{B} B_{i}}=\overrightarrow{O P}_{j}+{\overrightarrow{P_{j} B_{j}}}_{\text {(for } i=1,2,3} \\
& j=2,3,1) \\
& \left\|\overrightarrow{B_{i} B_{j}}\right\|^{2}=(\sqrt{3} r)^{2}=\left\|\overrightarrow{O P_{j}}+\overrightarrow{P_{j} B_{j}}-\overrightarrow{O P_{i}}-{\overrightarrow{P_{i} B_{i}}}\right\|^{2}
\end{aligned}
$$

From the above (5), the implicit relationships between $\theta_{i}$ and six lengths of the extensible links $L_{\mathrm{i}}, S_{\mathrm{i}}$ are described as follows:

$$
\begin{aligned}
& f_{1}\left(\theta_{1}, \theta_{2}\right)=L_{1}{ }^{2}+L_{2}{ }^{2}+L_{1} L_{2} \cos \theta_{1} \cos \theta_{2}-2 L_{1} L_{2} \sin \theta_{1} \sin \theta_{2} \\
& -L_{1} \cos \theta_{1}\left(3 R+\sqrt{3} S_{2}\right)+L_{2} \cos \theta_{2}\left(-3 R+\sqrt{3} S_{1}\right)+3 R^{2}-3 r^{2} \\
& +S_{1}{ }^{2}+S_{2}{ }^{2}+S_{1} S_{2}+R\left(-\sqrt{3} S_{1}+\sqrt{3} S_{2}\right)=0 \\
& f_{2}\left(\theta_{2}, \theta_{3}\right)=L_{2}{ }^{2}+L_{3}{ }^{2}+L_{2} L_{3} \cos \theta_{2} \cos \theta_{3}-2 L_{2} L_{3} \sin \theta_{2} \sin \theta_{3} \\
& -L_{2} \cos \theta_{2}\left(3 R+\sqrt{3} S_{3}\right)+L_{3} \cos \theta_{3}\left(-3 R+\sqrt{3} S_{3}\right)+3 R^{2} \\
& -3 r^{2}+S_{2}{ }^{2}+S_{3}{ }^{2}+S_{2} S_{3}+R\left(-\sqrt{3} S_{2}+\sqrt{3} S_{3}\right)=0 \\
& f_{3}\left(\theta_{1}, \theta_{3}\right)=L_{1}{ }^{2}+L_{3}{ }^{2}+L_{1} L_{3} \cos \theta_{1} \cos \theta_{3}-2 L_{1} L_{3} \sin \theta_{1} \sin \theta_{3} \\
& -L_{1} \cos \theta_{1}\left(3 R-\sqrt{3} S_{3}\right)-L_{3} \cos \theta_{3}\left(3 R+\sqrt{3} S_{1}\right)+3 R^{2} \\
& -3 r^{2}+S_{1}{ }^{2}+S_{3}{ }^{2}+S_{1} S_{3}+R\left(\sqrt{3} S_{1}-\sqrt{3} S_{3}\right)=0
\end{aligned}
$$

We thus have three nonlinear equations for three unknowns $\theta_{i}$. Their solutions can be obtained numerically. To control the manipulator in real-time without additional sensor for sensing the position and orientation of the end effector installed at the top plate, it is very important to compute $\theta_{i}$ fast by using $L_{\mathrm{i}}$ and $S_{\mathrm{i}}$ sensed from the position sensors of the links. In this paper, the Newton-Raphson method is used to solve the nonlinear equation. Since the initial value of $\theta_{i}$ is critical for the calculation time of the Newton-Raphson method, the initial value has to be guaranteed to meet the required computing time constraints within the workspace. Then, the position of the center $\left(X_{\mathrm{c}}, Y_{\mathrm{c}}, Z_{\mathrm{c}}\right)$ and the orientation vector $(\vec{n}, \vec{a}, \vec{a})$ of the top plate are obtained from the calculated $\theta_{i}$ as follows:

$$
\begin{aligned}
& X_{C}=\frac{1}{3}\left(\frac{\sqrt{3}}{2} S_{3}-\frac{\sqrt{3}}{2} S_{2}-L_{1} \cos \theta_{1}+\frac{1}{2} L_{2} \cos \theta_{2}+\frac{1}{2} L_{3} \cos \theta_{3}\right) \\
& Y_{C}=\frac{1}{3}\left(S_{1}-\frac{1}{2} S_{2}-\frac{1}{2} S_{3}+\frac{\sqrt{3}}{2} L_{2} \cos \theta_{2}+\frac{\sqrt{3}}{2} L_{3} \cos \theta_{3}\right) \\
& Z_{C}=\frac{1}{3}\left(L_{1} \sin \theta_{1}+L_{2} \sin \theta_{2}+L_{3} \sin \theta_{3}\right) \\
& {[\vec{n}, \vec{o}, \vec{a}]=\left[\begin{array}{lll}
n_{1} & a_{1} & a_{1} \\
n_{2} & o_{2} & a_{2} \\
n_{3} & a_{3} & a_{3}
\end{array}\right]=} \\
& {\left[\begin{array}{ccc}
\frac{R-L_{1} \cos \vartheta_{1}-X_{C}}{r} & \frac{\sqrt{3} S_{2}+L_{2} \cos \vartheta_{2}-L_{1} \sin \vartheta_{2}-3 X_{C}}{\sqrt{3} r} & m_{2} O_{3}-m_{3} O_{2} \\
\frac{S_{1}-Y_{C}}{r} & \frac{\sqrt{3} R+S_{1}-S_{2}-\sqrt{3} L_{2} \cos \vartheta_{2}-3 I_{C}}{\sqrt{3} r} & n_{3} q_{1}-m_{1} O_{3} \\
\frac{L_{1} \sin \vartheta_{1}-Z_{C}}{r} & \frac{L_{1} \sin \vartheta_{1}+2 L_{2} \sin \vartheta_{2}-3 Z_{C}}{\sqrt{3} r} & n_{1} \vartheta_{2}-m_{2} O_{1}
\end{array}\right]}
\end{aligned}
$$

where $\vec{a}=\vec{n} \times \vec{o}$.

From (6), we know that multiple solutions of the angles $\theta_{i}$ exist for a given set of link lengths. In other words, there are multiple possible configurations of the manipulator for a specific set of link displacements.

\section{Existence of the Forward Kinematic Solution}

Since a parallel manipulator consists of closed chains, there are kinematic constraints that restrict the motion of the links of the manipulator. Some solutions of the direct kinematics are not realizable according to combinations of the link displacements. In this section, we introduce conditions for checking the existence of the solution of the direct kinematics for the proposed in-parallel manipulator.

The kinematic constraints that restrcit the motion of the links are described by (6). From the constraints, we determine the critical points that has an unique kinematic solution in the whole workspace. A set of equations for obtaining the points at which the rates of variation of $f_{1}, f_{2}$, and $f_{3}$ are zero can be derived as follows: 


$$
\begin{aligned}
& \frac{\partial f_{1}}{\partial \theta_{1}}+\frac{\partial f_{1}}{\partial \theta_{2}}=0 \\
& \frac{\partial f_{2}}{\partial \theta_{2}}+\frac{\partial f_{2}}{\partial \theta_{3}}=0 \\
& \frac{\partial f_{3}}{\partial \theta_{1}}+\frac{\partial f_{3}}{\partial \theta_{3}}=0 .
\end{aligned}
$$

Let us denote

$$
\rho=\frac{r}{R}
$$

In consideing the configuration of the proposed mechanism, we know that the solutions simultaneously satisfying (9) are only the following two cases:

$$
\begin{array}{ll}
\theta_{1}=\theta_{2}=\theta_{3}=0 & \text { if } \rho=\frac{r}{R} \leq 1, \\
\theta_{1}=\theta_{2}=\theta_{3}=\pi & \text { if } \rho=\frac{r}{R}>1 .
\end{array}
$$

The above two cases represents the configurations of the mechanism whose forward kinematic solution exists uniquely. Therefore, the existence condition of the forward kinemation solution including the critical conditions of (11) can be described as follows:

$$
\begin{array}{ll}
f_{i}(0,0) \leq 0 & \text { if } \rho=\frac{r}{R} \leq 1, \\
f_{i}(\pi, \pi) \leq 0 & \text { if } \rho=\frac{r}{R}>1, \quad \text { for } i=1,2,3 .
\end{array}
$$

Let's consider a special case in which the lengths of three vertical links and three horizontal links are same respectively: $L_{1}=L_{2}=L_{3}=L, S_{1}=S_{2}=S_{3}=S$. In this case, the solutions satisfying the inequality of (12) are given in forms as:

$$
\begin{array}{lll}
L \geq R-\sqrt{r^{2}-S^{2}} & \text { if } \rho=\frac{r}{R} \leq 1 \quad(-r \leq S \leq r), \\
L \geq\left|-R+\sqrt{r^{2}-S^{2}}\right| & \text { if } \left.\rho=\frac{r}{R}\right\rangle 1 \quad(-r \leq S \leq r) .
\end{array}
$$

Fig. 3 shows the existence range of the forward kinematic solution satisfying the inequality of (13). For each $\rho$, (13) means that the kinematic solution extst at the upper zones of the obtained curves.

Generally, the forward kinematics of parallel manipulators doesn't have a unique solution, but multiple solutions. The simulations for the forward kinematics of the proposed manipulator result in eight distinct solutions according to the initially guessed $\theta_{i}$. Fig. 4 shows the results of (4) at the conditions which $R$ $=r=50[\mathrm{~mm}], L_{1}=L_{2}=L_{3}=100[\mathrm{~mm}], S_{1}=S_{2}=S_{3}=0$, and $-180^{\circ} \leq \theta_{i} \leq 180^{\circ}$ for $i=1,2,3$. We know that sets of $\left(\theta_{1}, \theta_{2}\right),\left(\theta_{2}, \theta_{3}\right)$, and $\left(\theta_{3}, \theta_{1}\right)$ satisfying form a certain shape of band respectively. These shapes mean that there exist multiple solutions for the forward kinematics of the proposed mechanism.

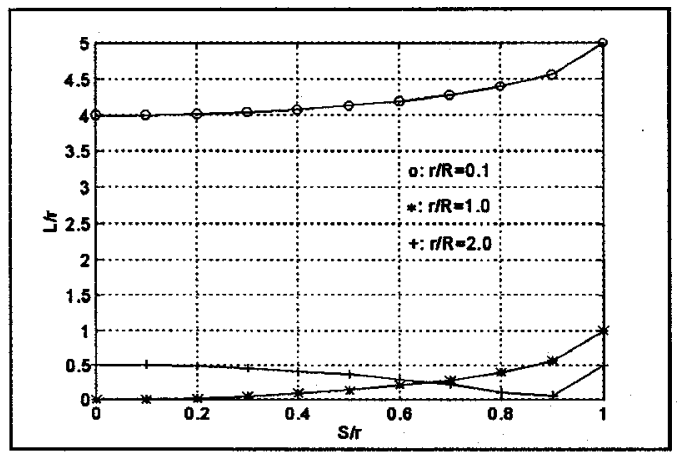

Fig. 3. Existence range of the forward kinematic solution

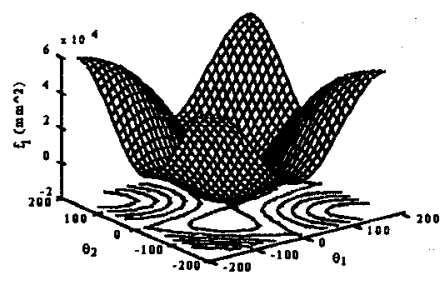

(a) $f_{1}\left(\theta_{1}, \theta_{2}\right)=0$

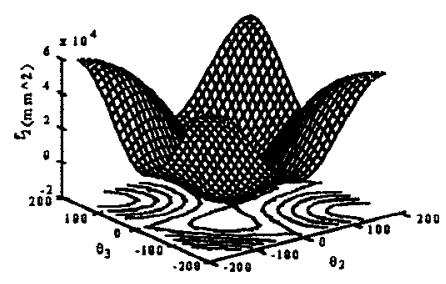

(b) $f_{2}\left(\theta_{2}, \theta_{3}\right)=0$

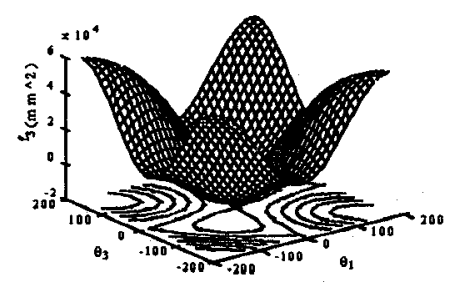

(c) $f_{3}\left(\theta_{3}, \theta_{1}\right)=0$

Fig. 4. Graphs of multiple existence of the forward kinematic solution

A series of numerical analysis represent the existence of the eight distinct forward kinematic solutions as shown in Fig. 5.

As noted earlier, fast computation of kinematics is very important to control a parallel manipulator in real 
time. In this paper, we developed the kinematic model on a personal computer with a 80486-DX2 66 MHz CPU. The inverse and forward kinematics take approximately 2 miliseconds. These results are fast enough to allow us to implement a real-time control of the proposed manipulator with a good frequency response.

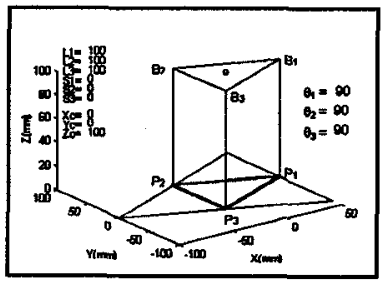

(a)

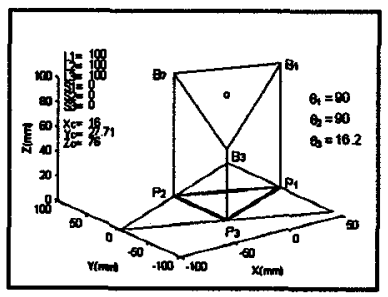

(c)

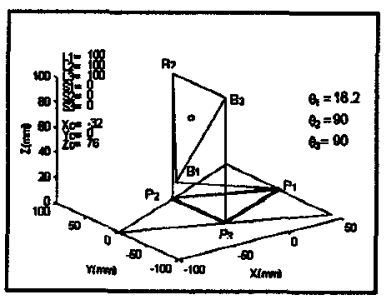

(e)

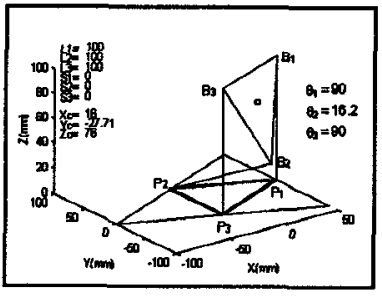

(g)

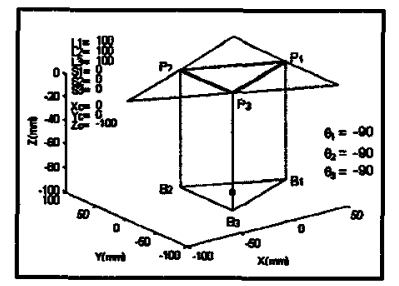

(b)

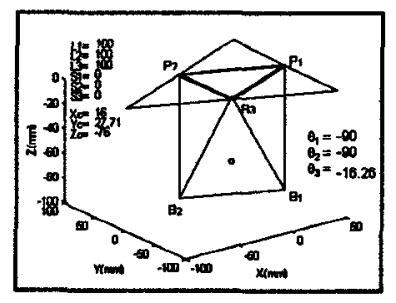

(d)

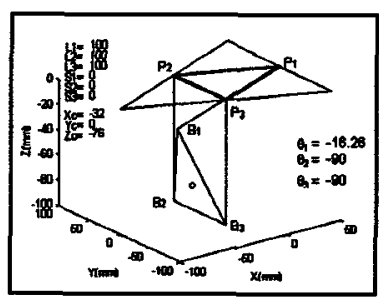

(f)

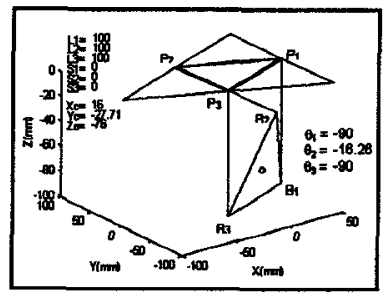

(h)
Fig. 5. Configurations of eight distinct forward kinematic solutions

\section{Work Space Analysis}

Generally, the work space of the Stewart platform type manipulator forms a certain type of an umbrellar whose sectional area is changing according to the height[14]. This characteristics may restrict the possible applications of the platform for various tasks. The proposed manipulator has an uniform workspace without variation of the sectional area, although its height varies. The workspace always forms a shape of a hexagonal pole irrespective of the variation of the ratio $\rho$. Fig. 6 shows the comparison of the workspaces of the two manipulators with $R=50$ [mm], $\rho=1,0 \leq L_{i} \leq 1.2 R$, and $-0.1 R \leq S_{i} \leq 0.1 R$ for $i=1,2,3$.

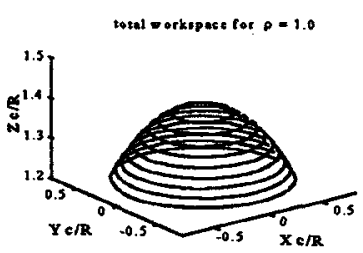

(a) Stewart platform

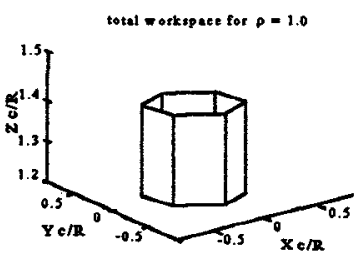

(b) the proposed manpulator

Fig. 6. Comparison of the workspaces for the Stewart platform and the proposed manipulator in case of $\rho=1$

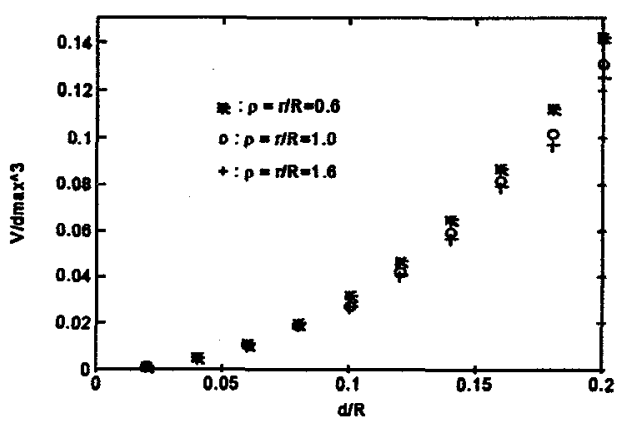

Fig. 7. Variation of workspace volume by varying the actuating lengths of the links $d$ and the $\rho$

The relationshiop between $\rho$ and the volume of the workspace by varying the actuating lengths of the links is shown in Fig. 7. Clearly, as $\rho$ decreses, larger workspace is obtained.

\section{Manipulability and Resistivity}

Manipulability measure $w$ was proposed to measure quantitatively the ability to change the position and orientation of the end-effector from the view point of the kinematics[13]. Since Jacobian matrix $J$ of parallel manipulators is derived from the inverse kinematics, it is the inverse one of the serial ones as follows: 


$$
F=\left[\begin{array}{c}
f_{c} \\
\tau_{c}
\end{array}\right]=J^{T} \tau
$$

where $f_{o}, \tau_{c}$ are the force and moment of the end-effector, respectively.

As the proposed manipulator has no redundant degree-of-freedom, $w$ is represented as follows:

$$
w=\left|\operatorname{det}\left(J^{-1}\right)\right| \text {. }
$$

The concept of resistivity measure, $w_{\mathrm{R}}$, of robot manipulators was suggested as a quantitative measure of their ability in resisting the externally applied forces[13]. The resistivity measure for the proposed manipulator can be written as follows:

$$
w_{\mathrm{R}}=\frac{1}{w}=\frac{1}{\left|\operatorname{det}\left(J^{-1}\right)\right|} .
$$

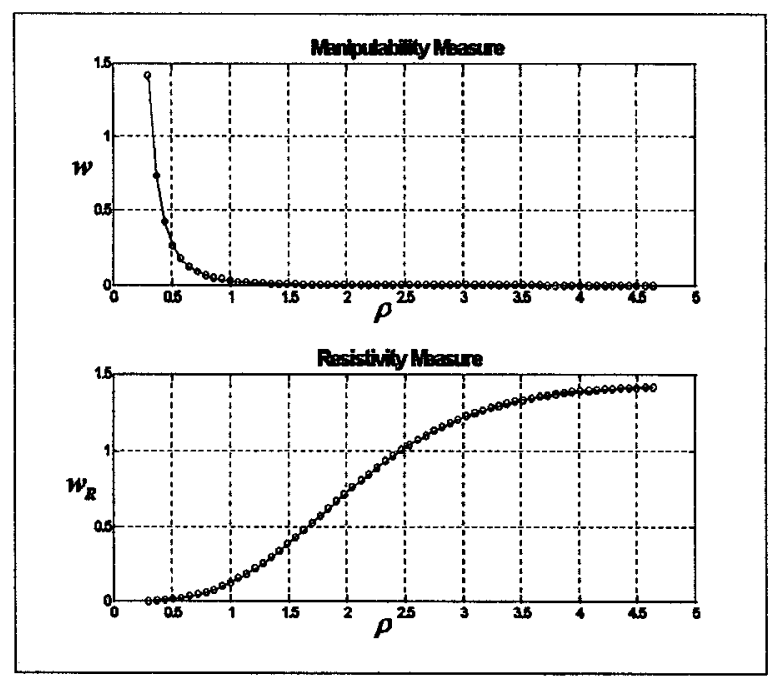

Fig. 8. Manipulability and resistivity with respect to $\rho$

Since the manipulability and resistivity are functions of the Jacobian, their values are changed according to configuration of the manipulator. For a configuration with $X_{\mathrm{c}}=0, Y_{\mathrm{c}}=0, Z_{\mathrm{c}}=60[\mathrm{~mm}]$ and the orientation angles of the top platform $\alpha=0^{\circ}, \beta=5^{\circ}, \gamma=0^{\circ}$, the simulation results by varying $\rho$ are shown in Fig. 8 .

It is found from the results of Fig. 8 that $\rho$ less than 1 results in rapid increase of the manipulability, while $\rho$ greater than 1 results in monotonously decreasing magnitude of the manipulability. But, the resistivity shows the reverse trends compared with the case of the manipulability. Therefore, the manipulator has to be designed as $\rho<1$ for good manipulation. But for large stiffness, it must be designed as $\rho>1$.

\section{Design of the Proposed Mechanism}

We developed a prototype of the proposed mechanism based on the design method described above. The mechanism will be used as a wrist of a robot for the probing task. The probing task requires large workspace, free manipulation, and high capability of resisting applied forces. In considering design tradeoffs among abovely discussed workspace, manipulability, and resistivity, it is decided that $\rho$ would be unity to compromise such considerations. The specification of the designed mechanism are $R=r=15$ [mm], $S_{i}=5$ [mm] and $L_{i}=90 \pm 2.5[\mathrm{~mm}]$ for $i=1,2,3$. In addition, these specification satisfy the condition of the existence of the forward kinematic solution.

Each joint actuator for the proposed machanism utilizes the Lorentz force: the force generated by a current-carrying conductor in a static magnetic field as shown in Fig. 9. The designed actuator has various features as a fast response, a minimal mass, and a linearizable force generation[12]. The measued force in motion direction of the actuator $0.8 \mathrm{~N} / \mathrm{A}$. The position of the actutor is measured by an optical sensor. This sensor is composed of a diode laser, two mirrors and a PSD( position sensing device). The position sensing resolution of this device is approximately $\pm 5 \mu \mathrm{m}$. To reflect accurately the applied forces on the links of the mechanism, an one dimensional force sensor is installed at each actuating link. The force sensor provided reading upto $10 \mathrm{~N}$ with a resolution of $2.22 \times 10^{-4} \mathrm{~N}$.

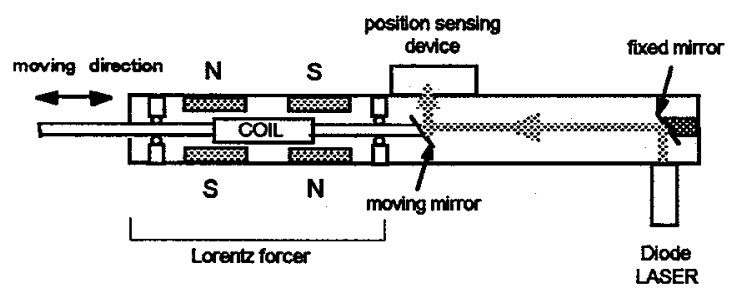

Fig. 9. The joint actuator with an optical sensing mechanism

\section{Conclusions}

We have presented a kinematic analysis of the developing 3 PRPS type parallel manipulator for the probing task application. The conditions for existence of the forward kinematic solutions are defined in terms of link displacements. The quantity $\rho$ can be used as a design parameter for determining the link lengths satisfying the conditions. The forward kinematic 
solution is not uniquely determined and eight distinct solutions exist according to the initially guessed quantities $\theta_{\mathrm{L}}$ The developed manipulator has the workspace which forms the shape of the hexagonal pole without change of the sectional area. $\rho$ (the ratio of the top plate and the base plate size) is also an improtant design parameter for determining the workspace volume. We designed a parallel wrist that has a desired workspace, manipulability, and resistivity for the probing task, and built it as a prototype. Each joint actuator for the proposed mechanism is an electromagnetic linear actuator that has a fast response and a linearized force generation suitable for the probing task. Future research will be directed towards the experimental investigation for active contact control.

\section{References}

[1] D. Stewart, "A Platform with Six Degree of Freedom", Proc. Inst. Mech. Eng., Vol. 180, No. 15, pp. 371-386, 1965.

[2] J.P. Merlet, "Force-Feedback Control of Parallel Manipulators", Proc. of IEEE Int. Conf. on Robotics and Automation, pp. 1484-1489, 1988.

[3] Y.K. Byun, D.Y. Kim, and H.S. Cho, "Analysis of a 6-DOF Pose/Wrench Sensor Integrated Stewart Platform-Based Robotic Wrist", Proc. of '95 ISMCR, pp.283-288, 1995.

[4] Z. Geng and L.S. Haynes, "Six-Degree-of-Freedom Active Vibration Isolation Using a Stewart Platform Mechanism", J. of Robotics Systems, Vol. 10, No. 5, pp. 725-744, 1993.

[5] C.L. Collins and G.L. Long, "The Singularity of an In-Parallel Hand Controller for Force-Reflected Teleoperation", IEEE Trans. on Robotics and Automation, Vol. 11, No. 5, pp.661-669, 1995.

[6] F. Behi, "Kinematic Analysis for a Six-Degree-ofFreedom 3-PRPS Parallel Manipulator", IEEE J. of Robotics and Automation, Vol. 4, No. 5, pp. 561-565, 1988.

[7] J. Hudgens and D. Tesar, "A Fully-Parallel Six Degree-of-Freedom Micromanipulator: Kinematic Analysis and Dynamic Model", Proc. 20th Biennial ASME Mechanisms Conf., Vol. 15-3, pp. 29-38, 1988.

[8] R.I. Alizade and N.R. Tagiyev, "A Forward and Reverse Displacement Analysis of a 6-DOF In-Parallel Manipulator", Mech. Mach. Theory, Vol. 29, No. 1, pp. 115-124, 1994.

[9] A. Sharon, N.N. Hogan, and D.E. Hardt, "High Bandwidth Force Regulatin and Inertia Reduction using a Macro/Micro Manipulator System", IEEE Int. Conf. on Robotics and Automation, pp. 126-132, 1988.

[10] C. Reboulet and R. Pigeyre, "Hybrid Control of A
6-DOF In-Parallel Actuated Micro-Manipulator Mounted on a Scara Robot", Int. J. of Robotics and Automation, Vol. 7, No. 1, 1992.

[11] J.H. Shim, H.S. Cho, and S. Kim, "Impact Control of a Probing Manipulator Contacting with Plastically Deformable Environment", Proc. of the 11th Int. Conf. on Systems Engineering, pp. 987-993, 1996.

[12] J.H. Shim, H.S. Cho, and S. Kim, "A New Probing System for the In-Circuit Test of a PCB", Proc. of the IEEE Int. Conf. on Robotics and Automation, pp. 580585, 1996.

[13] T. Yoshikawa, "Manipulability of Robotic Mechanism", Int. J. of Robotics Research, Vol. 4, No. 2, pp. 3-9, 1985.

[14] R.S. Stoughton and T. Arai, "A Modified Platform Manipulator with Improved Dexterity", IEEE Trans. on Robotics and Automation, Vol. 9, No. 2, pp. 166-173, 1993. 